\title{
Journal of Clinical and Nursing Research
}

\author{
Research Article
}

\section{The Influence of Let-7b-5p Target Gene in Cholangiocarc- inoma and its Bioinformatic Analysis}

\author{
Xibing Zhang ${ }^{1}$, Jianghua Ran ${ }^{1 *}$, Fang Liu ${ }^{2 *}$, Yingpeng Zhao ${ }^{1}$, Zongqiang $\mathrm{Hu}^{1}$ \\ ${ }^{1}$ Department of Hepatopancreatobiliary Surgery, The First Hospital of Kunming, Organ Transplantation Institute of Yunnan \\ Province, Yunnan Province, China \\ ${ }^{2}$ Department of Endocrine, The First Hospital of Kunming, Kunming 650000, Yunnan Province, China
}

Funding: Open project of Yunnan Provincial Organ Transplantation Clinical Medicine Center, 2020SYZ-Z-012.

\begin{abstract}
Objective: To analyze the biological functions and its clinical significance of let-7b-5p target gene in cholangiocarcinoma utilizing bioinformatics. Methods: The paper focuses on the let-7b-5p target gene, and predicts its biological functions as well as related signal pathways through GO biological function and KEGG signal pathway enrichment analysis. The STRING database and Cytoscape are used to construct a protein-protein interaction network to screen core genes. Results: The results of GO analysis showed that let-7b-5p target gene was mainly enriched in biological processes such as Small GTPase binding, Rho GTPase binding, and Rac GTPase binding. The results of KEGG analysis showed that let-7b-5p target gene was significantly enriched in key signaling pathways including Focal adhesion and ECM-receptor interaction. Through protein-protein interaction network and module analysis, CXCL8 and SDC2 were screened as the core site. Conclusion: let-7b-5p can participate in the regulation of biological functions of tumor cells in cholangiocarcinoma, suggesting that it may play an important role as a tumor suppressor gene and biomarker in the occurrence and development of cholangiocarcinoma, which provides new ideas for its diagnosis and treatment.
\end{abstract}

Keywords: Let-7b-5p; Cholangiocarcinoma; Bioinformatics; CXCL8; SDC2

Publication date: March, 2021

Publication online: 31 March, 2021

"Corresponding author: Jianghua Ran, Fang Liu, 329315398@qq.com
Cholangiocarcinoma (CCA) is a highly malignant bile tract tumor that originates from the epithelial cells of the inner lining of the bile duct. CCA accounts for about $5-10 \%$ of gastrointestinal tumors, which is more common in Asian countries. In the past 30 years, a number of studies have shown that the incidence and mortality of CCA are increasing globally ${ }^{[1]}$. Due to the hidden symptoms, the lack of specific diagnostic indicators, the difficulty of diagnosis, as well as biological characteristics of CCA, the prognosis is extremely poor. Although surgical resection has always been considered as the only potential way to cure CCA, the postoperative prognosis of patients is still poor, with only a 5-year survival due to some studies ${ }^{[2]}$. Therefore, there is an urgent need to find some molecular targets and tumor markers for early diagnosis and effective individualized treatment. MicroRNA is a type of endogenous noncoding single-stranded RNA molecule with a length of about 22 nucleotides, which can specifically bind to mRNA at the post-transcriptional level to regulate the expression of target genes, and plays a crucial role in the occurrence and development of tumors ${ }^{[3]}$. Studies have shown that let- $7 b-5 p$ is involved in the occurrence and development of liver cancer,esophageal cancer and so on.However, the specific mechanism and clinical significance of let$7 b-5 p$ in CCA have not been revealed yet ${ }^{[4-5]}$. With the development of bioinformatics, the improvement of many disease databases provide a theoretical basis for revealing the pathogenesis of human diseases and new therapeutic targets. The paper uses bioinformatic 
technology to screen out the differentially expressed genes (target genes) of CCA and their functions as well as signal pathways, and further excavate the core genes, so as to study the biological functions of let$7 b-5 p$ target gene and provide data and theoretical guidance.

\section{Materials and methods}

\subsection{The acquisition of let-7b-5p target gene}

The miRWALK database was used to identify 57943 binding sites with let- $7 b-5 p$, then screened with 3-UTR, 7245 sites were left.

\subsection{The GO enrichment analysis and KEGG pathway analysis of differential genes}

The DAVID database (https://david.ncifcrf.gov/) was used to analyze the selected target genes, and gene function annotation enrichment analysis was performed based on Gene Ontology (GO). Moreover, data was analyzed through biological processes (BP), cellular components (CC), and molecular function (MF). The Kyoto Encyclopedia of Genes and Genomes (KEGG) annotates target genes with a list of genes and pathway networks involved in it, and deals with genomes, cells, diseases, as well as signal pathways. In the paper, we analyzed the KEGG signal pathway through the KOBAS database, and the screening condition was $P<0.05$.

\subsection{The protein-protein interaction network analysis of differential genes}

The protein-protein interaction network (PPI) of differential genes and modular analysis were conducted through STRING database (http:// string-db.org/) and Cytoscape. The target gene was imported into the STRING database to perform PPI analysis. Then, use the degree plug-in of Cytoscape software to perform module analysis, and mine the most closely connected modules in the PPI to predict the interaction between the encoding proteins of the target genes. At the same time, screen out the most critical genes.

\section{Results}

2.1 The GO enrichment analysis and KEGG pathway analysis of let-7b-5p differential genes

The DAVID database was used to perform GO biological function enrichment analysis of target genes. In the biological process, these target genes are mainly involved in the activation of $\mathrm{T}$ cells and the reaction to acidic chemicals. In terms of cell components, target genes are mainly enriched in the apical plasma membrane and other parts. As for molecular functions, target genes are mainly enriched in Small GTPase binding, Rho GTPase binding, and Rac GTPase binding, etc, as shown in Figure 1. Enrichment analysis of KEGG signaling pathway suggested that significant target genes were mainly enriched in Focal adhesions and ECMreceptor interactions, as shown in Figure 2. The results of GO functional biological function and KEGG signal pathway enrichment analysis indicated that the biological function related to CCA was Focal adhesion and ECM-receptor interaction.

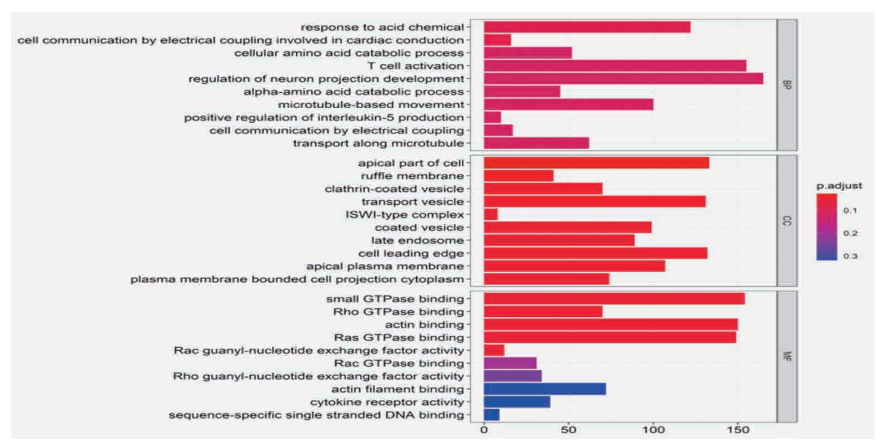

Figure 1. The GO biological function enrichment analysis of target genes

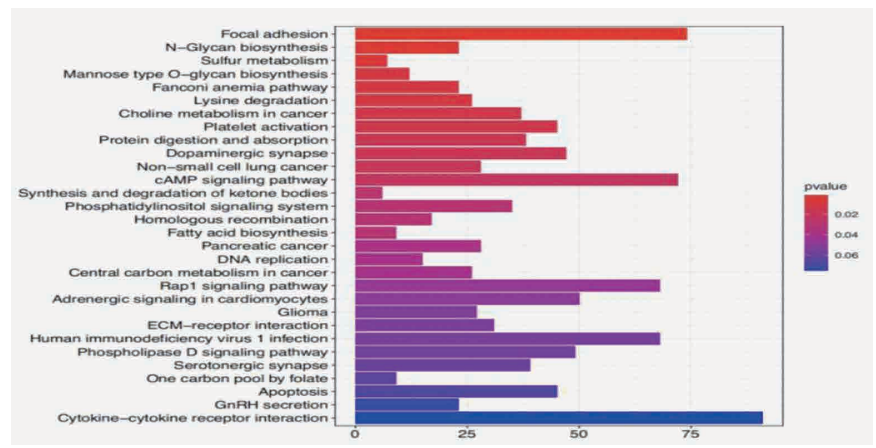

Figure 2. The KEGG signal pathway analysis of target genes

\subsection{Screening of key genes in the let-7b-5p protein-protein interaction network}

In order to screen out the differential genes that are highly related to let-7b-5p, we used the STRING database and Cytoscape to construct the proteinprotein interaction network, and screened out the top 10 key genes with the most obvious difference, as shown in Figure 3, which are CXCL8, GNG4, GNG2, HTR1A, SDC2, APOB, DRD2, CCL13, ACKR3, 
and TAS2R7, respectively. The results showed that CXCL8 and SDC2 proteins are closely related to other proteins.

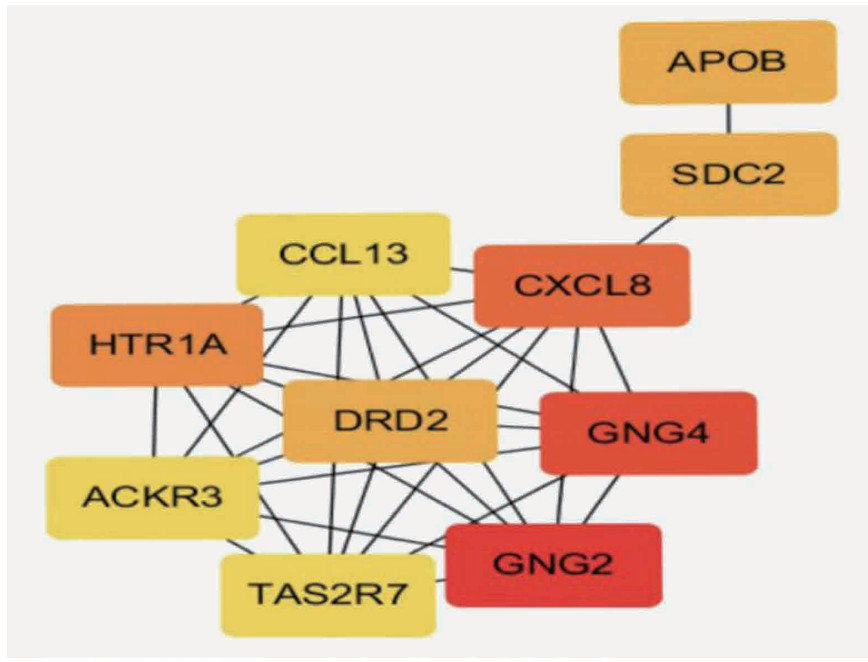

Figure 3. The diagram of protein-protein interaction network of key genes (The darker the color, the higher the ranking)

\section{Discussion}

Micro RNA is a type of non-coding single-stranded RNA molecules that is relatively conserved in evolution.Mature miRNA and Argonaute protein form an RNA-induced silencing complex, and can perform specific binding with the principle of complementary base pairing with the 3 'or 5 'end of the non-coding region of the target gene, and silence the target gene by inducing degradation of the target gene or inhibiting its translation, which indicates that miRNA can directly and accurately manage the expression of genes and function changes in cells ${ }^{[6]}$. Studies have shown that there are many types of miRNA target proteins, which play an important role in the process of cell proliferation, apoptosis, metabolism, and differentiation ${ }^{[6-8]}$. At present, miRNA has become a hot issue in the global field of tumor biology, as it plays an important role in the regulation of CCA. MiRNA with imbalanced expression can be detected in the tissues, blood, urine and bile of patients with CCA, which can be used as biological targets for the early diagnosis of cholangiocarcinoma.

The Let-7 gene cluster is a large family of miRNAs.They can maintain cell homeostasis and target oncogenes as well as other genes at the same time. In many types of human tumors, it can inhibit or interfere with the expression of downstream oncogenes to inhibit cancer ${ }^{[9]}$.
Let- $7 b-5 p$ belongs to the let-7, which has also been reported to play an important role in regulating the cycle and differentiation of cells as a tumor suppressor gene in a variety of cancers ${ }^{[4]}$. In liver cancer, SNHG16 promotes the cell cycle transition of G2/M by directly acting on the let-7b-5p axis, and promotes cell metastasis and EMT progression through regulating the let-7b-5p/HMGA2 axis in liver cancer ${ }^{[4]}$.In esophageal squamous cell carcinoma, let7b-5p can completely target KIAA 1377 and directly act on KYSE-150 and HeLa cells, and complete the regulation of inhibiting the proliferation, migration and invasion of cells ${ }^{[5]}$. However, the function of let$7 b-5 p$ in CCA is not fully understood. Few studies have shown that the expression level of Let-7b-5p in the plasma of patients with cholangiocarcinoma is significantly lower than that in normal patients, and there is a certain correlation between let$7 b-5 p$ and characteristics of clinical cases with cholangiocarcinoma, which is consistent with our previous research that the expression of let-7b-5p in cholangiocarcinoma tissues is lower than that of normal tissues. The specificity of let- $7 b-5 p$ plays an important role in the cells of all kinds of cancer, completing various functions such as the growth, differentiation, apoptosis and metabolism of cells, and may also affect the proliferation, apoptosis, migration and invasion of cells in CCA.

With the development of high-throughput sequencing technology and gene chips, the use of bioinformatics for deep analysis provides more methods for revealing the pathogenesis of human diseases and seeking new therapeutic targets. Let$7 b-5 p$ target genes were obtained from the gene database, and a total of 10 differentially expressed genes were screened for the most significant, namely CXCL8, GNG4, GNG2, HTR1A, SDC2, APOB, DRD2, CCL13, ACKR3, and TAS2R7. Proteinprotein interaction network suggested that CXCL8 and SDC2 gene-coded proteins were closely related to other proteins, thus playing an important role in the pathogenesis of CCA. CXCL8 is a member of cysteine-X-cysteine (CXC) chemokines, which is a multifunctional chemokine secreted by multiple types of cells. A large number of studies have proved that the abnormal level of CXCL8 in tumor cells promotes the occurrence and development of tumors, playing an important role in tumor growth, invasion, metastasis, angiogenesis and 
chemoresistance, activating signal pathways for the development of various human cancers ${ }^{[10-11]}$. Studies have found that $\mathrm{CAF}$ promotes the proliferation and invasion of cells in CCA, and Nintedanib inhibits the cancer-promoting effect of CAF by inhibiting CAF activation and CXCL8 secretion ${ }^{[12]}$. As an autocrine growth factor, CXCL8 is expressed higher in CCA than in normal tissues and is negatively correlated with the overall survival rate of patients, whose transduction has multiple effects on various types of cells in the tumor microenvironment. Targeting CXCL8 signal transduction is of great significance for delaying the progress of $\mathrm{CCA}^{[13]}$. The expression of Syndecan-2 (SDC2) is elevated in a variety of cancers, and is associated with progression and poor prognosis ${ }^{[14-15]}$. In addition, the expression of SDC2 is highly correlated with the progression and outcome of cholangiocarcinoma. It mediates cell adhesion and migration through multiple signal pathways such as syntenin-1, integrin $\alpha 2$ and RACK 1 , and enhances the synthesis of SDC2 through transforming growth factor- $\beta$ and melanocortin 1 receptors ${ }^{[16]}$. Moreover, SDC2 is a new target of PKM2, which plays an important role in the regulation of cell migration. Whether CXCL8 and SDC2 can be used as therapeutic targets or molecular diagnostic markers of CCA still needs further research.

GO enrichment analysis showed it was mainly enriched to the biological processes of Small GTPase binding, Rho GTPase binding, and Rac GTPase binding, which were related to the formation of adhesion plaques. Meanwhile, they can regulate the cytoskeleton, cell movement, and destroy cell polarity by regulating adhesion spots, and play an important role in the invasion and metastasis of malignant tumors ${ }^{[17-19]}$. In this study, the enrichment analysis of KEGG signaling pathway suggested that differentially expressed genes were mainly enriched in Focal adhesion (FA) pathway and ECM-receptor interaction pathway. (1) In the FA pathway, during cell migration, the FA is in a dynamic structure of assembly-disaggregation-reassembly. Its polarity distribution is consistent with the formation direction of lamellar pseudopodia, which requires the regulation of microfilament polymerization by Rac GTPase $^{[20]}$. Focal adhesions link actin skeletal fibers with ECM, which can transmit extracellular signals to the cytoplasm, providing power for cell migration and regulating cell adhesion and migration. The turnover of FA is a key link in the regulation of cell migration. The formation and turnover of FA are closely related to the main constituent protein FAK, and play a key role in regulating the turnover of $\mathrm{FA}^{[21]}$. The FAK signal generated by ECM-induced integrin aggregation is closely related to the cytoskeleton reorganization during the movement, which in turn shows that FAK can regulate cancer cells and promote the invasion of cancer cels ${ }^{[22]}$. A large number of studies have shown that FAK plays an important role in the occurrence, development and invasion of CCA, especially in the process of tumor invasion, as it regulates and mediates cancer signaling pathways. CCA not only affects the adhesion and proliferation of tumor cells, but also affects their adhesion and proliferation, which plays an important part in the process of angiogenesis ${ }^{[23]}$. (2) ECM-receptor interaction pathway: Extracellular matrix $(\mathrm{ECM})$ is composed of several macromolecules in a complex network, which mainly provides basic structural support, cell survival and suitable places, and affects many biological functions of cells by multiple signal pathways. The composition and organization of ECM are controlled by time and air conditioning, so as to control cell differentiation, but the imbalance of ECM dynamics and microenvironment can lead to tumors ${ }^{[24]}$. ECM plays a key role in regulating the progress and metastasis of CCA, which actively participates in the regulation of the growth, adhesion, invasion, migration and metabolic activities, especially on invasion and migration. It is mainly caused by the interaction between tumor cells and ECM specific molecules ${ }^{[25]}$. These studies support the conclusion of our study from many aspects, that is, let-7b-5p can regulate downstream target genes through Focal adhesion and ECM-receptor interaction signal pathways to participate in the occurrence and development of CCA.

In conclusion, the paper analyzed the differential expression and clinical significance of Let-7b-5p by bioinformatics, and constructed the regulatory network of Let-7b-5p target genes, analyzed its core proteins, then found it participate in the regulation of the biological functions of tumor cells in CCA, which may play an important role as the tumor suppressor gene and biomarker in the occurrence and development of cholangiocarcinoma, thus providing new directions and theoretical basis for studying CCA. 


\section{References}

[1] Khan AS, Dageforde LA. Cholangiocarcinoma[J]. SurgClin North Am, 2019, 99(2): 315-335.

[2] Yoh T,Seo S,Hatano E, et al. A novel biomarker-based preoperative prognostic grading system for predicting survival after surgery for intrahepatic cholangiocarcinoma [J]. Ann Surg Oncol, 2017, 24(5): 1351-1357.

[3] T, Volinia S, Calin G A, Liu CG, et al. A micro RNA expression signature of human solid tumors defines cancer gene targets [J]. Proc Natl Acad of Sci U S A, 2006, 103(7): 2257-2261.

[4] Li S,Peng F, Ning Y, et al. SNHG16 as the miRNA let-7b$5 p$ sponge facilitates the $\mathrm{G} 2 / \mathrm{M}$ and epithelial-mesenchymal transition by regulating $\mathrm{CDC} 25 \mathrm{~B}$ and HMGA2 expression in hepatocellular carcinoma [J]. Cell Biochem, 2020, 121(3): 2543-2558.

[5] Zheng S, Liu Q, Ma R, et al. Let-7b-5p inhibits proliferation and motility in squamous cell carcinoma cells through negative modulation of KIAA1377 [J]. Cell Biol Int, 2019, 43(6): 634641.

[6] Brennecke J, Hipfner D R,Stark A, et al. Bantam encodes a developmentally regulated micro RNA that controls cell proliferation and regulates the proapoptotic gene hid in Drosophila [J]. Cell, 2003, 113(1): 25-36.

[7] Chen X. A micro RNA as a translational repressor of APETALA2 in Arabidopsis flower development [J]. Science, 2004, 303(5666): 2022-2025.

[8] Xu P, Vernooy S Y, Guo M, et al.The Drosophila micro RNA Mir-14 suppresses cell death and is required for normal fat metabolism [J]. Current Biology, 2003, 13(9): 790-5.

[9] Ustianenko D, Chiu HS, Treiber T,et al. LIN28 selectively modulates a subclass of let-7 micro RNAs [J]. Mol Cell,2018, 71(2):271-283.

[10] Xie K. Interleukin-8 and human cancer biology [J]. Cytokine Growth Factor Rev., 2001,12(4): 375-91.

[11] Wang Y, Qu Y, Niu XL, et al. Autocrine production of interleukin-8 confers cisplatin and paclitaxel resistance in ovarian cancer cells [J]. Cytokine, 2011, 56(2): 365-75.

[12] Yamanaka T, Harimoto N, Yokobori T, et al. Nintedanib inhibits intrahepatic cholangiocarcinoma aggressiveness via suppression of cytokines extracted from activated cancerassociated fibroblasts [J]. Br J Cancer,2020, 122(7): 986-994.

[13] Sun Q, Li F, Sun F, et al. Interleukin-8 is a prognostic indicator in human hilar cholangiocarcinoma $[\mathrm{J}]$. Int J Clin
Exp Pathol, 2015, 8(7): 8376-84.

[14] Kim Y, Park H, Lim Y,et al. Decreased syndecan-2 expression correlates with trichostatin-A induced-morphological changes and reduced tumorigenic activity in colon carcinoma cells $[\mathrm{J}]$. Oncogene, 2003, 22(6): 826-30.

[15] Huang X, Xiao DW, Xu LY, et al. Prognostic significance of altered expression of SDC2 and CYR61 in esophageal squamous cell carcinoma [J]. Oncol Rep, 2009, 21(4): 11231129.

[16] Mytilinaiou M, Bano A, Nikitovic D, et al. Syndecan-2 is a key regulator of transforming growth factor beta2/Smad2mediated adhesion in fibrosarcoma cells [J]. IUBMB Life, 2013, 65(2):134-143.

[17] Clayton NS,Ridley AJ.Targeting Rho GTPase Signaling Networks in Cancer [J]. Front Cell Dev Biol, 2020, 8:222.

[18] Kazanietz MG, Caloca MJ. The Rac GTPase in cancer: From old concepts to new paradigms [J]. Cancer Res, 2017, 77(20): 5445-5451.

[19] Agochiya M, Brunton VG, Owens DW, et al. Increased dosage and amplification of the focal adhesion kinase gene in human cancer cells [J]. Onc ogene, 1999, 18(41): 5646-5653.

[20] Rikitake Y, Takai Y. Directional cell migration regulation by small G proteins, nectin-like molecule-5 and afadin [J]. Int Rev Cell Mol Biol, 2011,287:97-143.

[21] Kuo JC, Han XM, Hsiao CT, et al. Analysis of the myosin-IIresponsive focal adhesion proteome reveals a role for $\beta$-Pix in negative regulation of focal adhesion maturation [J]. Nat Cell Biol, 2011, 13(4): 383-93.

[22] Bouchard V, Demers M J, Thibodeau S, et al. Fak/Src signaling in human intestinal epithelial cell survival and anoikis: Differentiation state-specific uncoupling with the PI3-K/Akt-1and MEK/Erk pathways [J]. Journal of Cellular Physiology, 2010, 212(3): 717-728.

[23] Ohta R, Yamashita Y, Taketomi A,et al. Reduced expression of focal adhesion kinase in intrahepatic cholangiocarcinoma is associated with poor tumor differentiation $[\mathrm{J}]$. Oncology, 2006, 71(5-6): 417-22.

[24] Carpino G, Overi D, Melandro F, et al. Matrisome analysis of intrahepatic cholangiocarcinoma unveils a peculiar cancerassociated extracellular matrix structure $[\mathrm{J}]$. Clin Proteomics, 2019, 16:37.

[25] Brivio S, Cadamuro M, Strazzabosco M, et al. Tumor reactive stroma in cholangiocarcinoma: The fuel behind cancer aggressiveness [J]. World J Hepatol, 2017, 9(9): 455-468. 